\title{
Automatic detection of Sfe: a proposal
}

\author{
Juan José Curto, Santiago Marsal, Gastón Creci, and Gemma Domingo \\ Observatori de l'Ebre, (OE), CSIC, Universitat Ramon Llull, 43520 Roquetes, Spain \\ Correspondence to: Juan José Curto (jjcurto@obsebre.es)
}

Received: 21 February 2017 - Revised: 17 May 2017 - Accepted: 31 May 2017 - Published: 13 July 2017

\begin{abstract}
Solar flare effects (Sfe's) are rapid magnetic variations related to the enhancement of the amount of radiation produced during solar flare events. For several minutes, the ionosphere is activated and electron densities, electrical conductivities and electric currents are enhanced. The magnetic signature of a flare is visible in the illuminated hemisphere, and sometimes it shows up as small crochet-like movements in the magnetograms. However, regarding their detection using an automatized procedure, they have been very elusive because their small amplitude, which is close to the level of noise produced by other natural phenomena, and their highly variable irregular shapes make it extremely difficult to apply predesigned patterns. In this paper, we summarize the difficulties that Sfe detection presents and explain a line of work we initiated to overcome these difficulties with the goal of achieving a system capable of performing automatic detection. Some properties of Sfe's, including spherical symmetry around the vortex and different time durations between Sfe's and other natural variations, were used to construct an index allowing us to detect Sfe's.
\end{abstract}

Keywords. Geomagnetism and paleomagnetism (rapid time variations)

\section{Introduction}

Several rapid magnetic variations attracted the attention of scientists from an early moment. At the IAGA General Assembly (Rome, 1954) a new committee was created on rapid variations and Earth currents with A. Romañá from the Ebre Observatory elected as chairman. The task of the committee was to create lists of events, including sudden storm commencements (SSC), pulsations during sudden commencements (PSC), sudden impulses (SI) and solar flare effects (SFE), which were to be published in the IAGA annual bulletins (Curto et al., 2007). Since then, the working proce- dure to detect SSC and Sfe events has been more or less the same and it is still based on manual methods. Recently, many attempts to automatize the detection of sudden commencements (SC) have succeeded using different approaches, e.g., neural networks (Segarra and Curto, 2013), wavelet filters (Takano et al., 1999) and time-frequency clusters (Hafez and Ghamry, 2011). The key factor in the successful automatic detection of SSC was probably the fact that they mostly have a common morphological pattern: the main signal, a sudden increase in the $H$ component, is similar around the world. However, this is not true for Sfe's. They are elusive, so previous attempts at Sfe automatic detection failed and until now there have been no references in the scientific literature regarding their possible automatic detection.

\section{Difficulties in Sfe detection}

The first and most obvious difficulty in Sfe detection is that they do not have a definite morphological pattern. The Provisional Atlas of Rapid Variations (Romañá, 1959), which explains the first attempts at a systematic overview of the morphology of the Sfe, states: "It is almost impossible to give comprehensive examples of sfe, their aspect changing considerably with circumstances (magnetic or telluric component, moment of the diurnal variation, sun's altitude, intensity, etc.)". As a comment on some presented examples, it concludes: "But the following examples, taken also from cases confirmed by direct observation of the solar flare, have an aspect rather confusing". No one looking at only one single magnetogram can conclude whether a movement is or is not in fact a Sfe because this is only a partial view of the whole process (Curto and Gaya-Piqueé, 2009a, b). Sfe's can appear only in the $X$ magnetic component, only in the $Y$ magnetic component, in both components or in neither of them. Their shapes are irregular and unpredictable. Sometimes they are like a "crochet", but on other occasions they 

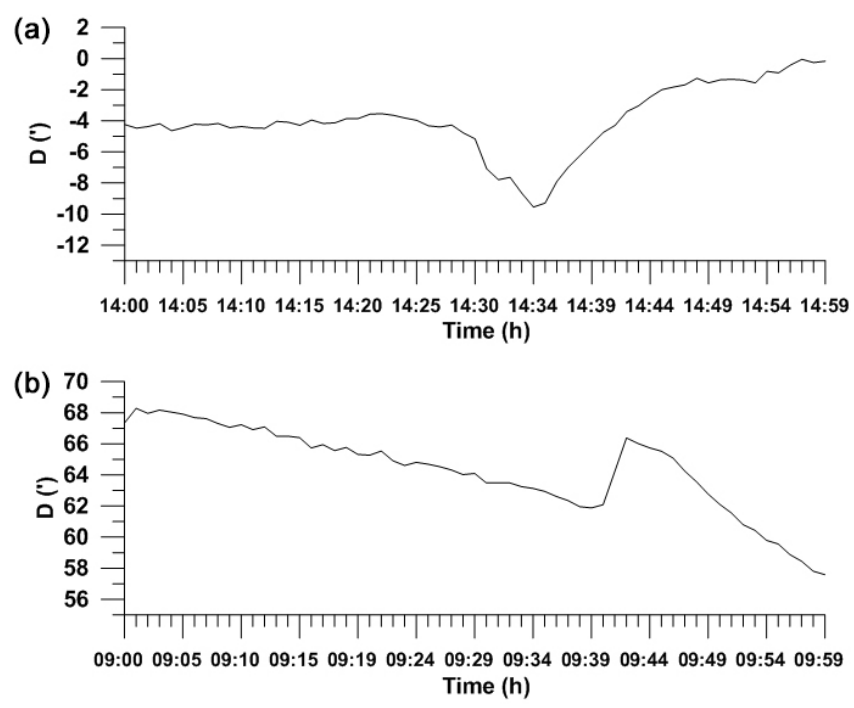

Figure 1. Two examples of Sfe's on the $D$ component at EBR magnetic observatory: (a) one at 14:34 UT on 9 March 2015 with a "V valley" shape and (b) the other at 09:42 UT on 21 June 2015 with a "crochet" shape.

look like a "V valley". Sometimes they are steep, but at other times they are smooth (Fig. 1). According to Curto et al. (2016b), this is due to the temporal variations in the radiation, which have irregular shapes in their origin: the solar disturbances. Moreover, Sfe events are the result of the action of several radiation bands ( $X$ and UV) that occur at different heights of the Sun, so they could be synchronic or not. They also have different relaxation times (Richmond and Venkateswaran, 1971). Thus, to achieve a certain degree of certainty in the Service of Rapid Magnetic Variations (Curto et al., 2007), other contemporary ionospheric effects are usually sought out (Tsurutani et al., 2009).

\section{A proposal for Sfe automatic detection}

\subsection{Spherical symmetry of the radiation}

Despite the abovementioned difficulties, there are some characteristics of Sfe phenomena that can be used as key elements to elucidate whether a movement in the magnetograms is or is not an effect of a solar disturbance. The symmetric distribution of the radiation around the subsolar point is one characteristic. Symmetric radiation means symmetric ionization and, to a certain extent, symmetric currents. These electric currents appear as a result of two systems that are not coincident in space: the first due to tidal winds moved by thermal cells because of the day-night temperature gradients and the second due to ionization induced by solar radiation, which is distributed as a spherical cap around the subsolar point. In any case, the resultant electric circuit has a circle-like shape flowing around a central point known as the current vortex.

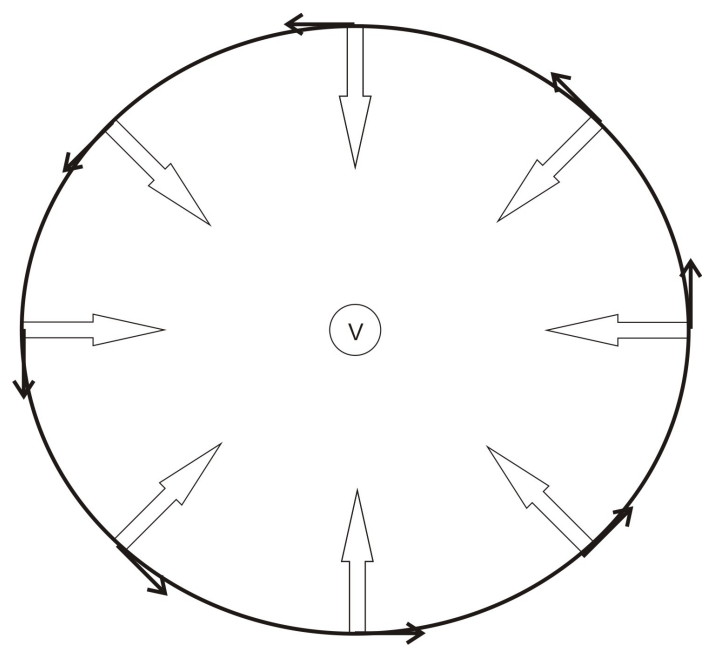

Figure 2. Simplified scheme of a circular system of electric currents like that of Sfe's in the Northern Hemisphere. Magnetic vectors point towards the vortex of the system.

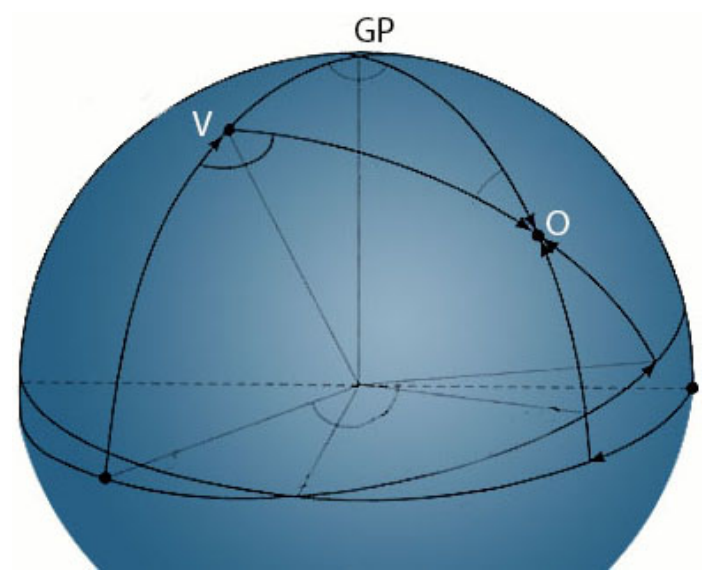

Figure 3. After the coordinate transformation, new coordinates can be computed for each magnetic observatory $(\mathrm{O})$. The new pole $(\mathrm{V})$ is the vortex of the current system instead of the geographic pole (GP).

Hence, the induced magnetic vectors point towards this center in the Northern Hemisphere (Fig. 2) and away from it in the Southern Hemisphere. By taking advantage of this, we can perform a coordinate transformation using the vortex point as the new origin of the coordinate system (center) instead of the geographic pole. We take the values given in Table 2 from Curto et al. (1994a) as the position of the current vortex where the seasonal variation in the Sfe vortex was computed using a parametric model. This table is the result of the analysis of 30 years of data. By solving the spherical triangle, we can obtain the new coordinates of the observatory (Fig. 3) and compute the radial component of the Sfe vector at a given observatory, i.e., its projection onto the direction given by the vortex-observatory line. By summing 


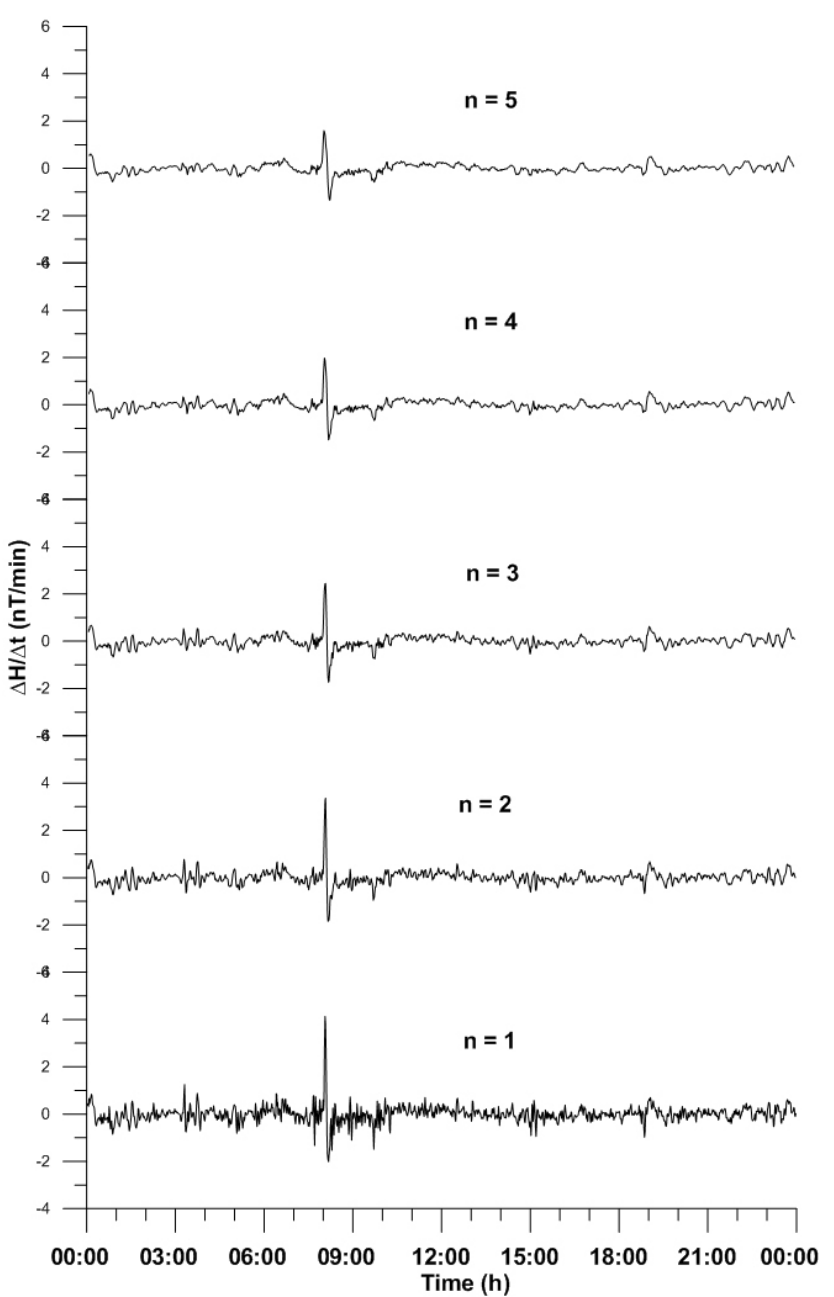

Figure 4. Derivate of the $H$ component for 9 August 2011 at Ebre Observatory acting as a high-pass filter. Increasing the width $(n)$ of the filter results in a reduction in the high-frequency noise, but the signal-to-noise ratio decreases more quickly. Thus, we chose $n=1$.

up the contributions of the whole group of observatories in the illuminated area, we can increase the signal-to-noise ratio. However, the perfect circular current model is a simplification of the reality. As shown by Curto et al. (1994a), the current vortex (loop) is usually elliptical with moderate eccentricities in solstices but achieving important eccentricities in equinox. The overall performance will necessarily be reduced due to the slight misalignment of the observatory signals outside the main axes of the ellipse.

However, manual operation is still necessary in this process to extract the $S_{q}$ contribution as a prior action before computing the Sfe vector. The diurnal magnetic variation, $S_{q}$, is always superposed on Sfe's and has a similar, although not identical, circular current system shape (Curto et al., 1994a). $S_{q}$ and Sfe current systems are not exactly coincident in space; generally in the Northern Hemisphere, the Sfe vortex is delayed from the $S_{q}$ vortex (Curto et al., 1994b). Thus,

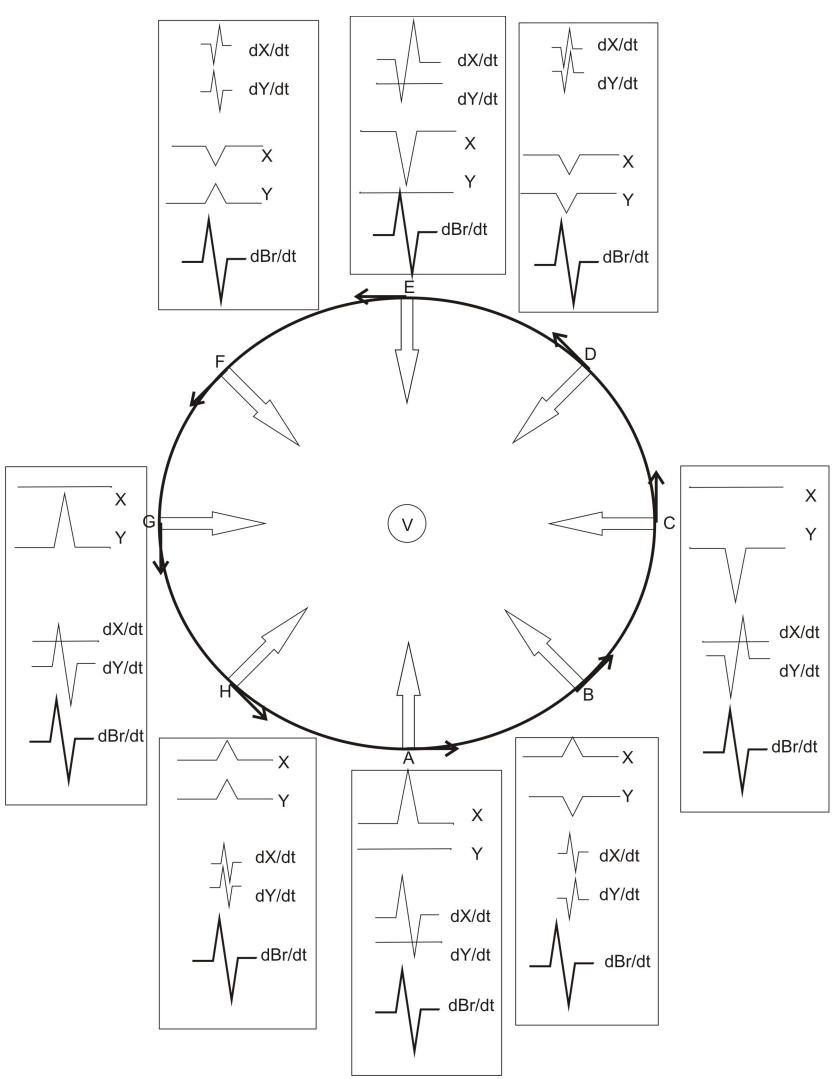

Figure 5. Sfe signatures from observatories under the electric current loop are very different depending on their location with respect to the vortex. However, after the coordinate transformation, the derivatives of the radial component of the Sfe vectors, $B_{\mathrm{r}}$, at different points of the circle $(\mathrm{A}-\mathrm{H})$ become coherent.

in each case, it has to be estimated manually. Observers estimate the diurnal variation trend by eye. However, a careless assessment of the $S_{q}$ level could mask the Sfe decay, which would translate directly into Sfe vector computation errors (Curto et al., 2016a). With the ideas proposed above we can improve the detection method but we will still not have achieved our goal, which is the complete automatization of the detection process.

\subsection{Different time durations}

From the above, we can conclude that the $S_{q}$ signal should be removed to produce an automatic process. To this end, we can take advantage of the different time durations of the signals. While $S_{q}$ variations last 1 day $\left(T_{S_{q}}=24 \mathrm{~h}\right)$, Sfe's usually last for several minutes $\left(T_{\mathrm{Sfe}}=0.2 \mathrm{~h}\right.$ ) (Curto et al., 1994a), so the frequency rate of $S_{q}$ is one hundredth that of a Sfe. With a similar approach as in electricity, we can use a high-pass filter to reject signals under the cutoff frequency. We will use the derivative as a digital high-pass filter. The derivative function is a very simple digital high-pass filter, and it is easy to implement. In fact, Sfe signals are usu- 


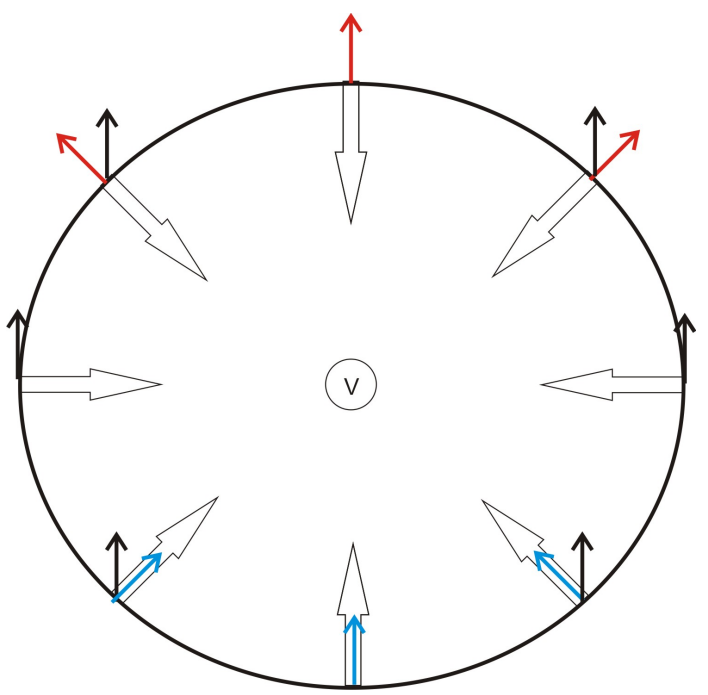

Figure 6. Signals from magnetic disturbances, like SC, cancel because the signal at one observatory is counterbalanced by the signal at its counterpart.

ally essentially a composition of step and ramp signals. After derivation, they become peaks and steps, respectively. We use a simple formulation:

$\dot{B}=\delta B(t) / \delta t=B(t+n \Delta t)-B(t-n \Delta t) / 2 n \Delta t$,

where $\dot{B}(t)$ is the time derivative of a magnetic field component, $\Delta t=1 \mathrm{~min}$ and $n$ controls the temporal width of the filter. In our case $n=1$ gives the best results (Fig. 4). This filter allows the Sfe signal to pass, but other natural signals with longer periods, like the $S_{q}$ signals, are attenuated.

\subsection{Sfe index}

With an appropriate coordinate transformation, which would mean transforming from a coordinate reference system based on the geographic pole to a coordinate reference system based on the current vortex pole, the problem becomes quite simple. Sfe variations occur mostly in the radial component, $B_{\mathrm{r}}$. The Sfe signals (and their derivatives) from the different observatories with plenty of different shapes are transformed into a unique and simple shape, thus becoming coherent signals (Fig. 5). In this way, multiplicity and complexity are transformed into singleness and simplicity.

At that point, a new index, $\delta \mathrm{SFE}_{T}$, can be defined as the mean of the derivatives of the radial components of the Sfe variations, $\Delta \dot{B}_{\mathrm{r}}$, of the considered observatories in the coordinate system with the vortex as a center:

$\delta \mathrm{SFE}_{T} \equiv\left(\Delta \dot{B}_{\mathrm{r} 1}+\Delta \dot{B}_{\mathrm{r} 2}+\ldots+\Delta \dot{B}_{\mathrm{r} n}\right) / n$,

where $n$ is the number of observatories considered. Regarding the signal-to-noise ratio, this index behaves in such a way that the Sfe signals are reinforced but other natural magnetic

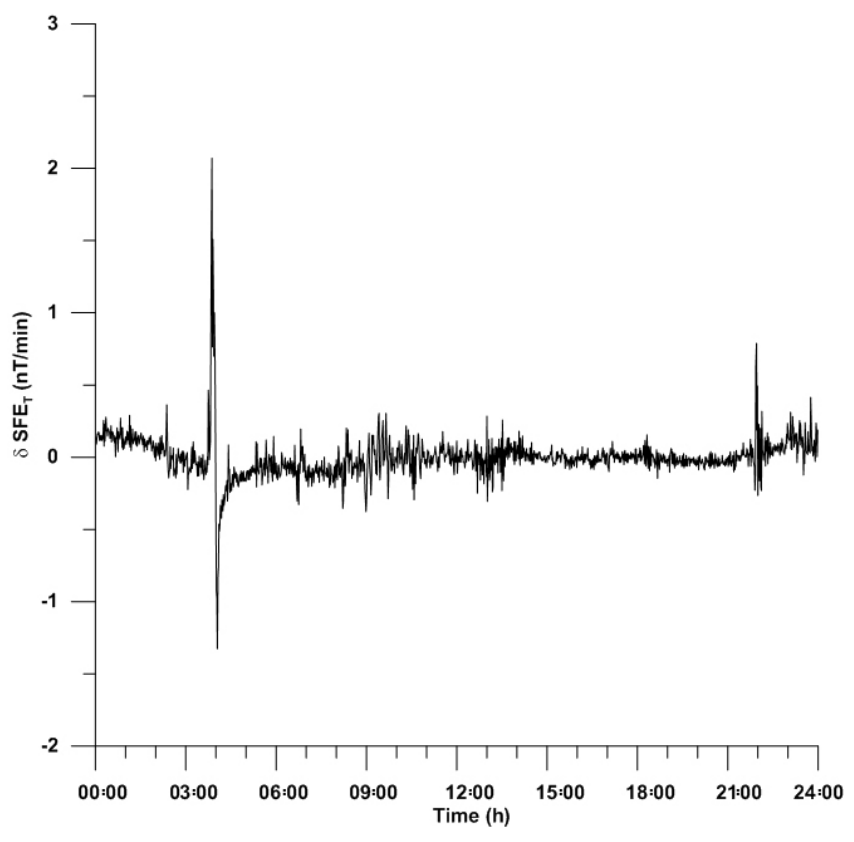

Figure 7. $\delta \mathrm{SFE}_{T}$ for 4 August 2011. On this day, Sfe's occurred at 03:44 UT and SSC occurred at 21:53. As shown, the algorithm shrinks the SC signal but cannot reject it completely.

disturbances (SC, pulsations or substorms), which could be considered as noise, become incoherent; in the mean, they almost cancel themselves out. Thus, the effect of SC, which are common rapid magnetic variations produced by the enhancement of the solar wind pressure, tend to vanish. When summing up the radial contributions of the observatories at a given longitude, the signals from observatories at a higher latitude with respect to the vortex are counterbalanced by the signals from observatories at a lower latitude (Fig. 6). To summarize, with this strategy we obtain a high commonmode rejection ratio, but the cancellation is not perfect. For example, SC disturbances have a latitude dependence, so low-latitude observatories have stronger signals than highlatitude observatories for the main phase (the step function), while the opposite is true for the preliminary impulses (PI; Araki, 1994; narrow sawtooth function). This produces an unavoidable certain level of noise. On the other hand, each observatory does not always have a counterpart observatory in the opposite part of the vortex. The lack of a counterpart observatory means that the SC signal is not counterbalanced. However, this undesirable effect is mostly shrunk by the mean when there is a large population of observatories available (Fig. 7).

Finally, a thresholding process is needed to separate Sfe signals from the remaining noise. We performed a statistical study to decide the appropriate thresholding value to best detect Sfe's. On the basis of a list of true Sfe events, we counted the number of false negatives as a function of the selected threshold value (Fig. 8). Note that, for a particular 


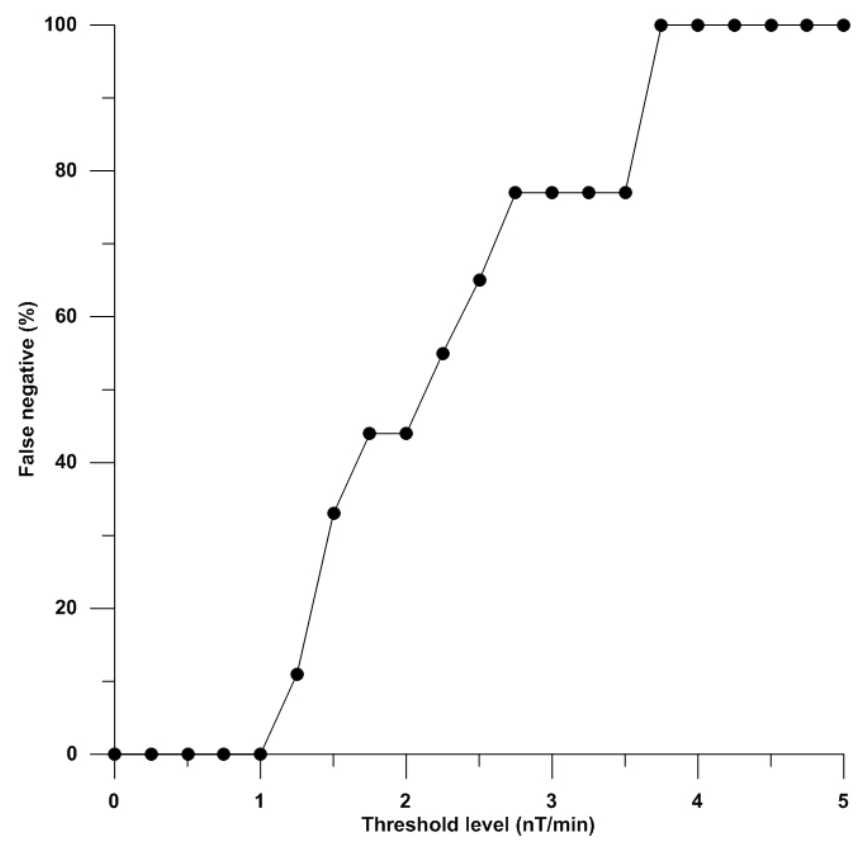

Figure 8. Percentage of false negatives as a function of the selected threshold value.

event, a given threshold is not considered a false negative if it succeeds in detecting the event at least at one point. We took $1 \mathrm{nT} \mathrm{min}-1$ as a threshold value to keep the clearest and biggest events.

An Sfe detector has been developed, and it is now working in a test mode with promising results. We work only on the lit hemisphere. In the first stage, we focus on the northern current loop because of the higher availability of magnetic observatories there; however, in a second stage, the southern current loop will also be analyzed for a comprehensive patrol. We analyze observatories at low and mid latitudes and exclude equatorial observatories because they are influenced by the equatorial electrojet. We also exclude high-latitude observatories because they are very influenced by auroral currents. The magnetic observatories used in each case vary according to the time of the event. Only those observatories in the lit area are taken. As an example of Sfe detection, we present an Sfe event that happened on 9 August 2011 (Fig. 9). This Sfe event was clearly identified through the Sfe index, $\delta \mathrm{SFE}_{T}$. A clear peak stands out above the noise level, providing easy detection after a thresholding process (Fig. 10).

\section{Conclusions}

Sfe detection is a difficult task because this kind of disturbance has a changing and unpredictable nature rather than regular patterns. This explains the absence of automatic detection methods so far. However, by taking advantage of some special characteristics of Sfe's according to their nature, including spherical symmetry around the vortex and dif-

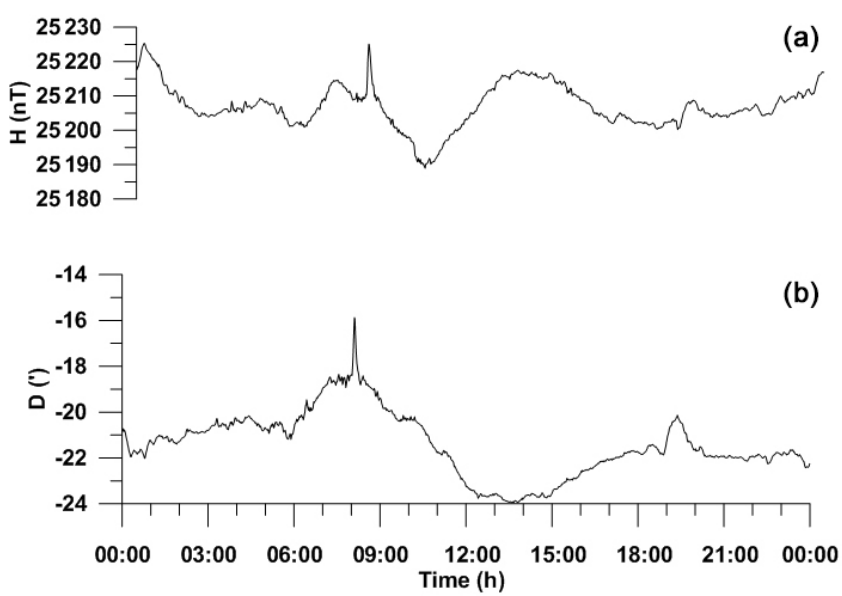

Figure 9. The Sfe as seen on the Ebre Observatory magnetogram in the $H$ and $D$ components at 08:00 UT on 9 August 2011.

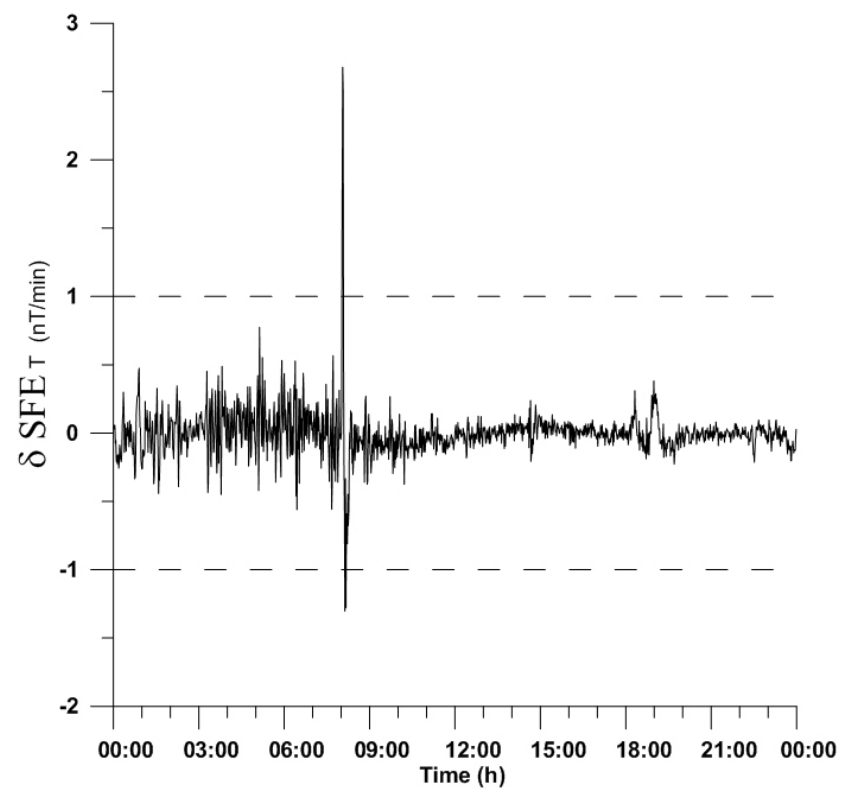

Figure 10. $\delta \mathrm{SFE}_{T}$ for 9 August 2011. A clear peak at 08:00 UT stands out from the noise, allowing for clear Sfe detection.

ferent time durations between Sfe's and other natural variations with similar spatial distributions like $S_{q}$, we were able to separate the Sfe signal from other natural disturbances. We defined an Sfe index by taking into account the derivative of the radial contribution of the Sfe disturbances with respect to the vortex of the current system responsible for the Sfe variation. This detector, now in a test phase, has proven successful in detecting Sfe events.

Data availability. Data used in this work came directly from their online Intermagnet database located at: http://www.intermagnet. org/. 
Competing interests. The authors declare that they have no conflict of interest.

Acknowledgements. This research has been supported by the Spanish government project CTM2014-52182-C3-1-P of MINECO. The authors also wish to thank INTERMAGNET and all the collaborating observatories providing high-quality magnetic data. Finally, we thank the International Service of Rapid Magnetic Variations and their collaborating observatories for providing lists of Sfe events.

The topical editor, Jean Rasson, thanks Christine AmoryMazaudier and one anonymous referee for help in evaluating this paper.

\section{References}

Araki, T.: A physical model of geomagnetic sudden commencement, AGU Geophys. Monogr., 81, 183-200, 1994.

Curto, J. J. and Gaya-Piqué, L. R.: Geoeffectiveness of Solar Flares in magnetic crochet (sfe) Production: I - Dependence on their spectral nature and position on the solar disk, J. Atmos. Sol.-Terr. Phy., 71, 1695-1704. https://doi.org/10.1016/j.jastp.2008.06.018, 2009a.

Curto, J. J. and Gaya-Piqué, L. R.: Geoeffectiveness of Solar Flares in magnetic crochet (sfe) Production: II - Dependence on the detection method, J. Atmos. Sol.-Terr. Phy., 71, 1705-1710, https://doi.org/10.1016/j.jastp.2007.12.003, 2009b.

Curto, J. J., Amory-Mazaudier, C., Cardús, J. O., Torta, J. M., and Menvielle, M.: Solar flare effects at Ebre: Regular and reversed solar flare effects, statistical analysis (1953 to 1985), a global case study and a model of elliptical ionospheric currents, J. Geophys. Res., 99, 3945-3954, 1994a.
Curto, J. J., Amory-Mazaudier, C., Cardús, J. O., Torta, J. M., and Menvielle, M.: Solar flare effects at Ebre: Unidimentional physical, integrated model, J. Geophys. Res., 99, 23289-23296, 1994b.

Curto, J. J., Cardús, J. O., Alberca, L. F., and Blanch, E.: Milestones of the IAGA International Service of Rapid Magnetic Variations, Earth Planets Space, 59, 463-471, 2007.

Curto, J. J., Castell, J., and Del Moral, F.: Sfe: waiting for the big one, Journal of Space Weather and Space Climate, 6, A23, https://doi.org/10.1051/swsc/2016018, 2016a.

Curto, J. J., Alberca, L. F., and Castell, J.: Dynamic aspects of the Solar Flare Effects and their impact in the detection procedure, J. Ind. Geophys. Union, 2, 99-104, 2016 b.

Hafez, A. G. and Ghamry, E.: Automatic detection of geomagnetic sudden commencements via time-frequency clusters, Adv. Space Res., 48, 1537-1544, 2011.

Richmond, A. D. and Venkateswaran, S. V.: Geomagneticcrochets and associated ionospheric current systems, Radio Sci., 6, 139164, 1971.

Romañá, A.: Provisional atlas of rapid variations, Annals of the International Geophysical Year, II B, 668-709, 1959.

Segarra, A. and Curto, J. J.: Automatic Detection of Sudden Commencements using Neural Networks, Earth Planets Space, 65, 791-797, https://doi.org/10.5047/eps.2012.12.011, 2013.

Takano, S., Minamoto, T., Arimura, H., Niijima, K., Iyemori, T., and Araki, T.: Automatic detection of geomagnetic sudden commencementsusing lifting wavelet filters, Lecture Notes in Computer Science, 1721, Springer, Berlin, Heidelberg, 1999.

Tsurutani, B. T., Verkhoglyadova, O. P., Mannucci, A. J., Lakhina, G. S., Li, G., and Zank, G. P.: A brief review of "solar flare effects" on the ionosphere, Radio Sci., 44, RS0A17, https://doi.org/10.1029/2008RS004029, 2009. 